\title{
A rare case report of advanced secondary abdominal pregnancy of 22 weeks during COVID 19 pandemic
}

\begin{abstract}
Abdominal ectopic pregnancy is a life-threatening condition where the embryo or foetus is growing in the abdominal cavity outside the uterus excluding fallopian tube, ovary or broad ligament. It can be primarily located in the peritoneal cavity or secondary to a ruptured ectopic pregnancy or tubal abortion. We are presentencing here a rare case of advanced abdominal pregnancy (AAP) in a very young women. Very few cases are reported till now and there are no clear cut guidelines regarding management of advanced abdominal pregnancy. This case we received when Covid 19 pandemic was as at its peak, many hospital were shut down by order of government authorities as not having the facility of following covid protocol regarding screening and management and elective surgeries were also abandoned in India. On ultrasound there was a foetus of 22 weeks found in mothers left lumber/left hypochondrium outside the uterine cavity. Her urgent laparotomy was planned as patient may land up in catastrophe any time and managed successfully with good co-ordination and support of various teams from Microbiology, Surgery, Anaesthesia, Obstetrics and Gynaecology departments of Sarojini Naidu Medical College Agra, Uttar Pradesh India. This case report supports the importance of routine antenatal checkups and first trimester ultrasound which was missed by patient that again may be because of lack of awareness and terrific environment because of pandemic. Timely diagnosis and teamwork management also plays important role for such a life-threatening condition to avoid maternal morbidity and mortality.
\end{abstract}

Keywords: ectopic pregnancy, advanced abdominal pregnancy, secondary abdominal pregnancy
Volume 5 Issue I - 202 I

\author{
Urvashi Verma, Asha, Meenal Jain, Prashant \\ Lawania, Shivani \\ Sarojini Naidu Medical College Agra, India
}

\author{
Correspondence: Urvashi Verma, Sarojini Naidu Medical \\ College Agra, 3/398, India, Tel 094I04I0383. \\ Email drurvashiverm@rediffmail.com
}

Received: October 30, 2020 | Published: Febrauary 08, 2021
Abbreviations: AAP, advanced abdominal pregnancy; POD, pouch of douglus

\section{Introduction}

Ectopic pregnancy is defined as pregnancy outside the normal uterine cavity. Rate of Ectopic pregnancy is about $1-2 \%$ of all pregnancies with $95 \%$ occurs in the fallopian tube. ${ }^{1}$ Incidence of Abdominal ectopic represent about $1 \%$ of all ectopic pregnancies. ${ }^{2}$ Pouch Of Douglus (POD) is most common location of abdominal pregnancy followed by mesosalpinx and omentum. However implantation on other abdominal organs such as spleen, liver and appendix is also reported. Advanced Abdominal pregnancy (AAP) is defined as pregnancy go beyond 20 weeks with signs of live foetus anywhere within the peritoneal cavity exclusive of tubal, ovarian or broad ligament. ${ }^{3}$ The Abdominal pregnancy is associated with higher Maternal mortality $0.5 \%$ to $18 \%$ and the perinatal mortality varies from 85 to $95 \%{ }^{4}$ Mortality is mainly due to the risk of massive haemorrhage from a partially or totally separated placenta at any stage of pregnancy. Diagnosis of abdominal pregnancy can be missed in low resources area with limited facilities and lack of awareness ${ }^{5}$ as in our case first scan was performed beyond 20 weeks. Diagnosis becomes difficult without ultrasound facility. Usually patient present with complications such as abdominal pain due to haemoperitoneum and diagnosed at the time of laparotomy. For the diagnosis of primary abdominal pregnancy Studdiford's criteria need to be filled. These include: (a) Normal bilateral fallopian tubes and ovaries (b) the absence of utero peritoneal fistula (c) pregnancy related exclusively to the peritoneal surface and early enough to eliminate the possibility of secondary implantation following a primary location in the tube. ${ }^{6}$ Advanced abdominal pregnancy is still rare, and guidelines for it's management are yet unclear with few cases published.

\section{Case report}

A 20 year old primigravida from rural area of Dholpur admitted in Gynaecology department of S.N. Medical College Agra on 31.5.2020 with the chief complaints of amenorrhoea for five months, pain in abdomen and loss of perception of fetal movement for one day. Patient was mild pale with normal blood pressure $110 / 70 \mathrm{mmHg}$ with tachycardia $104 / \mathrm{min}$, oxygen saturation was $98 \%$ on room air. On examination abdomen was distended with palpable foetal parts on right side of flank. Fetal heart sound was not detactable on fetal Doppler (Figure 1). Dull percussion with mild tenderness was also present. Uterine contour was not demarkable. On per vaginal examination cervix closed and bleeding was absent. Her investigation showed Haemogobin $7.7 \mathrm{gm} \%$, Total leucocyte Counts 11800, Differential leucocyte Counts- Polymorphs 86, Lymphocytes 10, Monocytes-01, Eosinofils 03 Serum Bilirubin $1.06 \mathrm{mg} / \mathrm{dl}$ SGOT 42.50 IU/L, SGPT 47.3IU/L, S. Ảlkaline Phosphatase 538.54 IU/L, Random Blood Sugar $66.2 \mathrm{mg} / \mathrm{dl}$, S. Urea 20.97, S. Creatinine $0.66 \mathrm{mg} / \mathrm{dl}$, S. Electrolytes were normal. Her urgent departmental ultrasound was done to confirm the diagnosis. It shows single dead foetus 21.4 weeks lying above the level of umbilical part (Figure 2). One Unit blood was transfused pre 
operatively. As patient was admitted at the COVID 19 pandemic so her urgent COVID sampling was done. Her consent was taken for laparotomy.

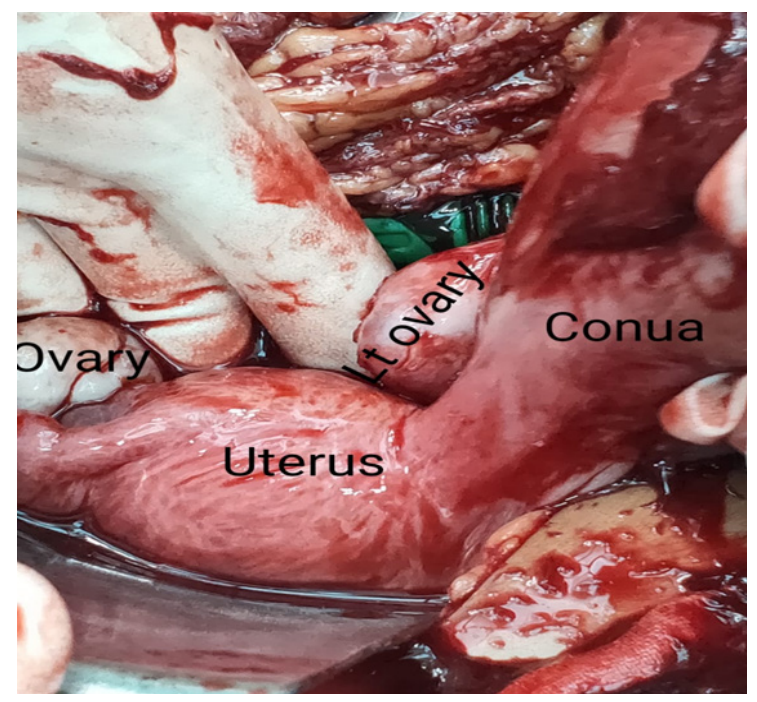

Figure I Fetal heart sound was not detactable on fetal Doppler.

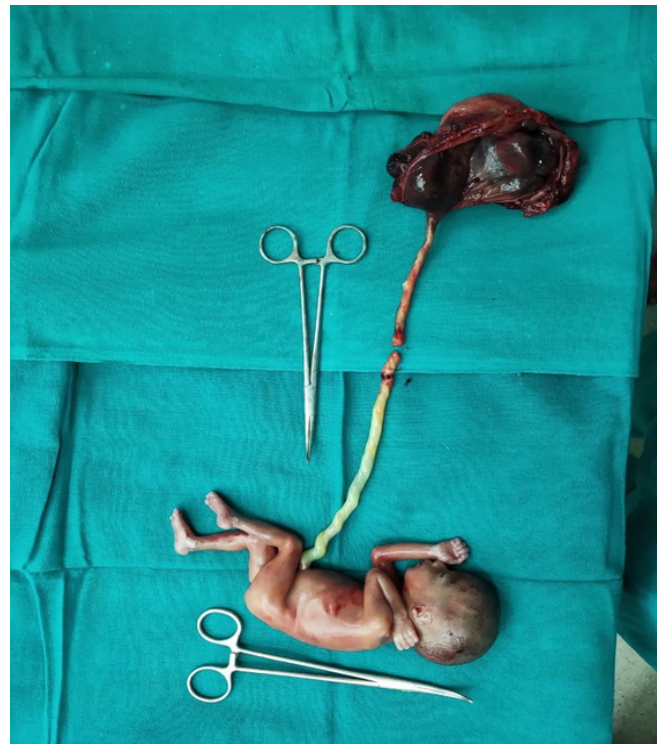

Figure 2 It shows single dead foetus 21.4 weeks lying above the level of umbilical part.

Epidural anaesthesia was given. 2 Unit blood was arranged and patient shifted to OT for laparotomy. Abdomen was opened hemoperitoneum found and $750 \mathrm{ml}$ of blood suctioned. A dead foetus was taken out from the right side of flank. Cord identified ligated and cut. Left cornua of uterus was found ruptured with some part of placenta in situ, rest of uterus was intact and normal in size and contour (Figure 3). Rent over cornua was sealed off by omentum. Part of Placenta found in abdominal cavity adhered to omentum and intestine. Bilateral ovaries were visualised and found normal. After removing foetus from abdominal cavity tried removal of placenta as much as possible, cornual resection was done along with bowel exploration to rule out placental invasion and injuries with proper haemostasis. Mild erosion and congestion were present over the colon and caecum part. Abdomen irrigation done with normal saline.
Abdomen closed in layers. Placenta and dissected tissue of cornual end with remaining placenta sent for histopathological examination. Patient shifted to ward in satisfactory condition. 2 Unit whole blood was transfused postoperatively. Patient orally allowed on $4^{\text {th }}$ postoperative day. Her post-operative period was uneventful. Her dressing was done and drain was also removed after 72 hour of surgery. Her complete stitches were removed on $8^{\text {th }}$ post-operative day. She discharged on same day in satisfactory condition.

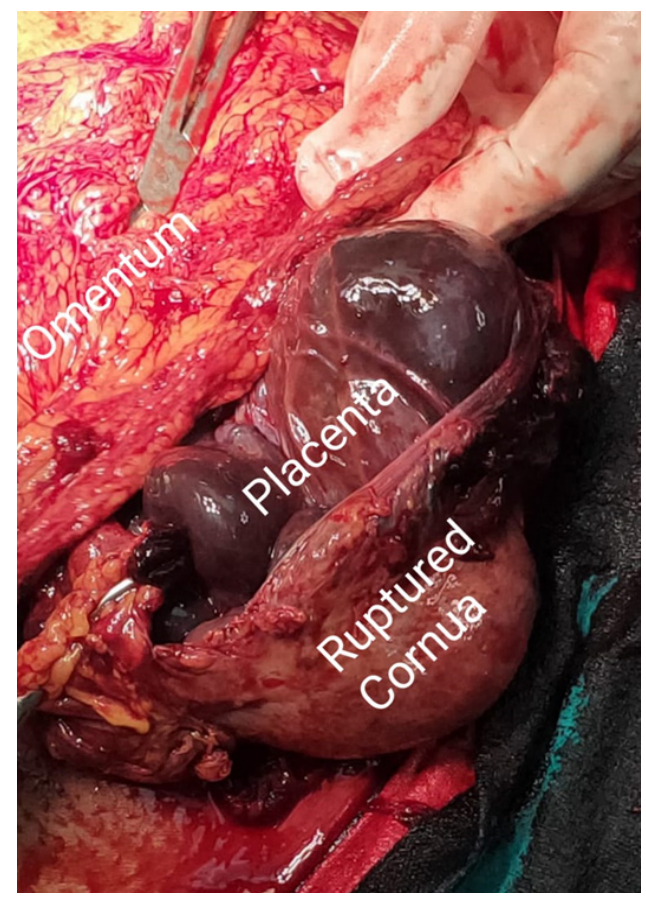

Figure 3 Left cornua of uterus was found ruptured with some part of placenta in situ, rest of uterus was intact and normal in size and contour.

\section{Discussion}

Abdominal pregnancy is highly uncommon. ${ }^{8}$ Abdominal pregnancy is classified as primary and secondary. Primary abdominal pregnancy is rarely seen. Secondary abdominal pregnancy is more common. Patient usually diagnosed on the basis of suspicion. The clinical presentation of an abdominal pregnancy can differ from that of a tubal pregnancy. ${ }^{9}$ Although there may be variability in the symptoms. Patient may be asymptomatic or may be present with severe abdominal pain, loss of foetal movements, foetal demise, easily palpable foetal parts. Early diagnosis and immediate treatment required in these cases. Ultrasound is very important in the diagnosis of extra-uterine pregnancy. MRI and CT scan is also important for diagnosis but they are not readily available. Lockhat et al. ${ }^{10}$ confirmed the value of MRI in the diagnosis of abdominal pregnancy. ${ }^{10}$ The risk factors are same as for tubal pregnancy. The recent use of progesterone only pills and intrauterine devices with a history of surgery, pelvic inflammatory disease, and allergy increases the risk of ectopic pregnancy. ${ }^{11}$ Our patient was not using any contraception and did not report any history of risk factors. She conceived spontaneously within a month of her marriage. Presence of placental tissue inside the cornua indicate that primarily it was cornual pregnancy that may be the reason of lesser peritoneal adhesions. Once abdominal pregnancy is diagnosed, laparotomy is usually treatment of choice to allow better assessment of placental attachment and to maintain all haemostasis. 
Current concepts on management of abdominal pregnancy support immediate active surgical intervention with termination of the pregnancy if diagnosed before 24 weeks gestation. ${ }^{11}$

\section{Conclusion}

Undiagnosed abdominal ectopic pregnancy in advanced stage is a little rare condition. Very few cases reported with advanced abdominal pregnancy till date. Termination of pregnancy is the mainstay of treatment in case of abdominal pregnancy. Failure to diagnose abdominal pregnancy can have fatal consequences. Ultrasound is still the procedure of choice for diagnosis of abdominal pregnancy. MRI can be used to confirm diagnosis, unfortunately these advanced imaging technologies are not readily available everywhere. ${ }^{12}$ In rare cases diagnosis can be missed if attention did not paid or if the radiologist is unexperienced. Early and accurate diagnosis plays important role in reducing mortality and morbidity.

\section{Declaration of patient consent}

The patient consent was taken for her images and clinical information to report in journal. All efforts were made to conceal her name and identity.

\section{Disclosure}

There are no conflicts of interest.

\section{References}

1. Sunita Dubey, Mohit Satodiya, Priyanka Garg. Abdominal pregnancy. A case report. Ann Afr Med. 2004;3(4):195-196.

2. Jurkovic D. Diagnosis and management of ectopic pregnancy. British Medical Journal. 2011;342:3397.
3. Worley KC, Hnat MD, Cunningham FG. Advanced extrauterine pregnancy: diagnostic and therapeautic challenges. Am J Obstet G ynecol. 2008;198(3):297.e1-7.

4. 4. Bright AS, Maser AH. Advanced abdominal pregnancy- review of the recent literature and report of a case. Obstetrics Gynecol. 1961;17:316324.

5. Sfar E, KabbarH, Marrakechi O, et al. Abdominal pregnancy, a rare anatomoclinical entity. 4 case reports. Rev Fr Gynecol Obstet. 1993;88(4):261265.

6. Parekh V.K, Bhatt S, Dogra VS. Abdominal pregnancy: an unusual presentation. J Ultrasound Med. 2008;27(4):679-681.

7. Amritha B, Sumangali T, Priya B, et al. A rare case of term viable secondary abdominal pregnancy following rupture of a rudimentary horn. A case report. J Med case reports. 2009;3:38.

8. Opare-Addo HS, Daganus S. Advanced abdominal pregnancy: a study of 13 consecutive cases seen in 1993 and 1994 at Komfo Anokye Teaching Hospital, Kumasi, Ghana. Afr J Reproductive Health. 2000;4(1):28-39.

9. Ad Rahman MS, Al Suleiman SA, Rahman J, et al. Advanced abdominal pregnancy- observation in 10 cases. Obstetrics Gynecol. 1982;59:366372.

10. Lockhat F, Corr P, Ramphal S, et al. The value of magnetic resonance imaging in the diagnosis and management of extra-uterine abdominal pregnancy. Clin Radiol. 2006;61:264-269.

11. Ankum WM, Mol BW, Van der Veen F, et al. Risk factors for ectopic pregnancy: a meta-analysis. FertilSteril. 1996;65(16):58320-58324.

12. Bertrand G, Le Ray C, Simand-Emond L, et al. Imaging in the management of abdominal pregnancy: a case report and review of the literature. Journal of Obstetrics Gynaecology Can. 2009;31(1):57-62. 